EESTI NSV TEADUSTE AKADEEMIA TOIMETISED. XVIII KÖIDE

KEEMIA * GEOLOOGIA. 1969, NR. 3

ИЗВЕСТИЯ АКАДЕМИИ НАУК ЭСТОНСКОИ ССР. ТОМ ХVIII

ХИМИЯ * ГЕОЛОГИЯ. 1969, 스 3

O. KIRRET, E. KULLIK

\title{
ANALYSE DER POLYURETHAN-ELASTOMERFASERN MITTELS DER PYROLYSE-GASCHROMATOGRAPHIE
}

Unter den neuen Errungenschaften auf dem Gebiet der synthetischen Fasern ist die Polyurethan-Elastomerfaser hervorzuheben, welche die Elastizität des Kautschuks besitzt.

Die Polyurethan-Elastomere sind Block-Copolymere. Im Netz ihrer Makromolekein finden sich weiche Segmente - amorphe Kettenabschnitte niedriger Polymerisationsstufe an kurze harte Kristallsegmente gebunden.

Sie werden aus leichtschmelzenden Makrodiolen synthetisiert, die sich durch eine monofunktionelle Additionsreaktion mit aromatischen Diisozyanaten in Makrodiisozyanate verwandeln. Im weiteren Gang der Reaktion entstehen aus den Makrodiisozyanaten durch blockweise Kettenverlängerung - mit niedrigmolekularen Diolen loder Diaminen behandelt - die Copolymere. Von den Makrodiolen werden Polyäther und Polyester mit Molekelmassen 1000 bis $4000\left[^{1}\right]$ benutzt.

Die Pyrolyse-Gaschromatographie hat bei der Lösung der verschiedensten Probleme Anwendung gefunden $\left[{ }^{2}\right]$, von denen die Analyse der polymeren Stoffe eine der Richtungen bildet. In einer Reihe von Fällen ist die Pyrolyse-Gaschromatographie für die Identifizierung der Polymere verwendet worden. So hat z. B. Cox 39 verschiedene polymere Stoffe pyrolysiert, die entweder fest, gummiähnlich oder von viskoser Konsistenz waren $\left[{ }^{3}\right]$. Desgleichen hat Voigt mit Identifizierungszwecken natürliche und synthetische Elastomere sowie einige Polymere pyrolysiert $\left.{ }^{4,5}\right]$. Näher sind die mit der Analyse von Polymeren und mit der Pyrolyse-Gaschromatographie verbundenen Fragen von Zulaica und Guiochon $\left[{ }^{6-8}\right]$ untersucht worden. Arbeiten von Küllik und Kirret $\left[{ }^{9-12}\right]$ behandeln ausführlicher die Möglichkeiten der Analyse natürlicher und chemischer Faserstoffe, wobei die verwendete Apparatur, die Analysebedingungen und -ergebnisse in Arbeit $\left[{ }^{11}\right]$ zu finden sind. Die Methode ermöglicht es, durch Vergleich der Chromatogramme der Pyrolyseprodukte mit den Etalonchromatogrammen und den relativen Retentionszeiten der Peaks die Faserart zu bestimmen. Waren die Fasern strukturell wenig untersucht wie z. B. Polyamid 6, Polyamid 6.6, Polyamide 7 und 11, so gab ihre Bestimmung auf Grund der Retentionszeiten der Peaks der Pyrolyseprodukte unbefriedigende Resultate, da die Chromatogramme überaus ähnlich aussahen. Die besten Resultate wurden erzielt, wenn bei der Identifizierung der Polyamide das Verhältnis des in einer Kieselgelsäule abgesonderten Methans und Kohlenstoffoxids gebraucht wurde $\left[{ }^{11}\right]$. Auch Haase und Rau $\left[{ }^{13}\right]$ haben die Möglichkeiten der pyrolytisch-gaschromatographischen Analyse der Faserstoffe betrachtet.

Aufgabe der vorliegenden Arbeit war es, solche Möglichkeiten für gleichartige, doch von verschiedenen Firmen hergestellte Fasern zu erforschen.

Vom Standpunkt des hierdurch aufgestellten Problems sind die Polyurethan-Elastomere (Tab. 1) von hohem Interesse.

Alle untersuchten Fasern waren weißfarbig und äußerlich betrachtet unterschiedslos. Bei der stereomikroskopischen Betrachtung konnten nur 
Tabelle 1

Verzeichnis der pyrolysierten Polyurethan-Elastomere

\begin{tabular}{c|ll}
\hline $\begin{array}{c}\text { Ordnungs- } \\
\text { nummer }\end{array}$ & Benennung des Faserstoffes & \\
\hline 1. & Spanzelle Elastomer & $140 \mathrm{den}$ \\
2. & Glanzstoffe Elastomer & Td 280 \\
3. & Dorlastan Bayer Elastomer & $140 \mathrm{den}$ \\
4. & Chemstrand Elastomer & $140 \mathrm{den}$ \\
5. & «Lycra» Elastomer & $140 \mathrm{den}$ \\
6. & Fabelta Fil. Elastomer & $420 \mathrm{den}$ \\
& & $48 \mathrm{TEX}$ \\
7. & Fr. elastomère & $140 \mathrm{den}$
\end{tabular}

minimale Unterschiede im Längsschnitt bemerkt werden, die mit der Technologie der Herstellung verbunden sind und nicht als charakteristische Differenzen gelten können.

Tabelle 2 bringt die Bedingungen der gaschromatographischen Analyse der Pyrolyseprodukte.

Arbeitsbedingungen der verwendeten Säulen

Tabelle 2

\begin{tabular}{|c|c|c|c|}
\hline \multirow[b]{2}{*}{ Arbeitsbedingungen } & \multicolumn{3}{|c|}{ Charakter des Fülimaterials } \\
\hline & $\begin{array}{l}\text { Kieselgel } \\
\text { (SG) }\end{array}$ & $\begin{array}{l}\text { 2,4-Dimethyl- } \\
\text { sulpholan (DMS) }\end{array}$ & $\begin{array}{l}\text { Polyethylenglykol } \\
1500 \text { (PEG 1500) }\end{array}$ \\
\hline Verwendete Säulen & Kupfer & $\begin{array}{l}\text { Rostfreies } \\
\text { Stahlrohr }\end{array}$ & $\begin{array}{l}\text { Rostfreies } \\
\text { Stahlrohr }\end{array}$ \\
\hline $\begin{array}{l}\text { Säulenlänge und innerer } \\
\text { Säulendurchmesser, cm } \\
\text { Füllmaterial }\end{array}$ & $\begin{array}{c}400 \times 0,39 \\
\text { Kieselgel }\end{array}$ & $\begin{array}{c}600 \times 0,39 \\
\text { Chromosorb } P \\
60 / 80 \text { mesh }\end{array}$ & $\begin{array}{l}350 \times 0,3 \\
\text { Chromosorb P } \\
60 / 80 \text { mesh }\end{array}$ \\
\hline $\begin{array}{l}\text { Verhältnis der festen und der } \\
\text { stationären Phase } \\
\text { Säulentemperatur }{ }^{\circ} \mathrm{C} \\
\text { Geschwindigkeit des Träger- } \\
\text { gases He, L/h }\end{array}$ & $\begin{array}{l}\overline{55} \\
3,8\end{array}$ & $\begin{array}{c}80 / 20 \\
42 \\
3,8\end{array}$ & $\begin{array}{c}80 / 20 \\
100 \\
3,8\end{array}$ \\
\hline
\end{tabular}

Die Fasern wurden vorher mit Äthylalkohol und Ather gereinigt. Die Polyurethan-Elastomere wurden bei der Temperatur von $500^{\circ} \mathrm{C}$ pyrolysiert. Diese Temperatur erwies sich bei vorangegangenen Forschungsarbeiten als die vom Standpunkt der qualitativen Analyse effektivste, da verzeichnet wurde, daß die bei $500^{\circ}-600^{\circ} \mathrm{C}$ pyrolysierte Faser am meisten charakteristische Pyrolyseprodukte gibt $\left[{ }^{10},{ }^{11}\right]$. Die analysierte Fasermenge war 2-$2,5 \mathrm{mg}$, die Dauer der Pyrolyse 10-12 sek. Für die Analyse der Pyrolyseprodukte wurde der Gaschromatograph YX-1 benutzt. Ein früher konstruiertes zusätzliches Pyrolysegerät erlaubte, die Zeit und Temperatur konstant und reproduzierbar zu halten $\left[{ }^{1 i}\right]$.

Die Pyrolyseprodukte der Fasern wurden mit drei verschiedenen Säulen analysiert, die folgendes absonderten: 1) die leichteren gasförmigen Produkte ( $\mathrm{CO}, \mathrm{CO}_{2}, \mathrm{CH}_{4}$ usw.), 2) die leichteren Kohlenwasserstoffe und 3) die leichteren Kohlenwasserstoffe mitsamt den ersten Gliedern der Alkohol- und Aldehydreihen.

Bei der Analyse der gasförmigen Kohlenwasserstoffe der Pyrolyseprodukte, in denen die Zahl der Kohlenstoffatome nicht größer ist als $\mathrm{C}_{5}$, erwies 
sich die Dimethylsulpholansäule (DMS) mit stationärer Phase als die beste $\left[{ }^{14}\right]$. Mit den anderen stationären Phasen verglichen, wie z. B. Apiezone, Silikonöle, Tweens, Squalan, $\beta, \beta^{\prime}$-Oxidipropionitril und verschiedenen Polyethylenglykolen erwies sich Polyethylenglykol 1500 (PEG 1500) bei der Lösung der vorliegenden Frage als das effektivste.

Die mit der Kieselgelsäule von den Pyrolyseprodukten abgesonderten leichteren gasförmigen Komponenten sind ihrem Chromatogramm nach bei allen Polyurethan-Elastomeren qualitativ ähnlich: überall sehen wir Peaks von $\mathrm{CO}, \mathrm{CH}_{4}, \mathrm{CO}_{2}$ und $\mathrm{C}_{2} \mathrm{H}_{6}$. Merkliche Unterschiede kommen aber im quantitativen Verhältnis der identifizierten Komponenten zum Vorschein. Auf Abb. 1 findet sich ein typisches mit der Kieselgelsäule erhaltenes Chromatogramm eines Polyurethan-Elastomers. Aus den Angaben der Tabelle 3 ersieht man, daß die behandelten Polyurethan-Elastomere bei der Pyrolyse in einer unterschiedlichen Art und Weise zerfallen.

Der größte Unterschied im CO-Gehalt der Pyrolyseprodukte ist bei Lycra und Fr. elastomère zu verzeichnen: in den Pyrolyseprodukten von Lycra gibt es Kohlenstoffoxid 4,36mal mehr als in denen von Fr. elastomère. Die übrigen pyrolysierten Fasern zeigen an CO-Gehalt Zwischenwerte. Bedeutend höher ist dieser Gehalt noch bei Glanzstoffe und Fabelta. Mit der Zunahme des CO-Gehalts wächst entsprechend auch der Gehalt an $\mathrm{CH}_{4}$ und $\mathrm{C}_{2} \mathrm{H}_{6}$. Dorlastan weist im Gehalt an $\mathrm{C}_{2} \mathrm{H}_{6}$ und Spanzelle im Gehalt an $\mathrm{CH}_{4}$ einige Abweichungen auf. Der Gehalt an Kohlenstoffdioxid dagegen nimmt in der Reihe Fr. elastomère - Lycra 1,9mal ab. Der Gehalt an Kohlenstoffdioxid fällt gleichzeitig von 85 auf $43,5 \%$. Auch verringert sich der summarische Gehalt an sauerstoffhaltigen leichteren gasförmigen Produkten $\left(\mathrm{CO}+\mathrm{CO}_{2}\right)$. Im allgemeinen können die auf der Grundlage ihrer Pyrolyseprodukte betrachteten Elastomere in zwei Gruppen eingeteilt werden:

1) Glanzstoffe, Fabelta und Lycra

2) Fr. elastomère, Spanzelle, Chemstrand und Dorlastan.

Tabelle 3

Resultate der pyrolytisch-gaschromatographischen Analyse und der Elementaranalyse von Polyurethan-Elastomeren

\begin{tabular}{|c|c|c|c|c|c|c|c|c|c|}
\hline \multirow[b]{2}{*}{ Faser } & \multicolumn{5}{|c|}{ Flächeninhalt d. Peaks $(\%)$} & \multicolumn{4}{|c|}{$\%$ der Elementaranalyse } \\
\hline & $\mathrm{CO}$ & $\mathrm{CH}_{4}$ & $\mathrm{C}_{2} \mathrm{H}_{6}$ & $\mathrm{CO}_{2}$ & $\underset{\mathrm{CO}_{2}}{\mathrm{CO}}$ & C & $\mathrm{H}$ & $\mathrm{N}$ & $\mathrm{O}$ \\
\hline $\begin{array}{l}\text { Fr. elastomère } \\
\text { Spanzelle } \\
\text { Chemstrand } \\
\text { Dorlastan } \\
\text { Glanzstoffe } \\
\text { Fabelta } \\
\text { Lycra } \\
\text { Polyurethan* }\end{array}$ & $\begin{array}{r}5,0 \\
7,2 \\
8,6 \\
9,1 \\
14,0 \\
19,9 \\
21,8\end{array}$ & $\begin{array}{r}8,3 \\
10,4 \\
9,4 \\
11,3 \\
12,6 \\
18,3 \\
25,3\end{array}$ & $\begin{array}{l}1,5 \\
1,8 \\
1,9 \\
2,8 \\
2,5 \\
3,3 \\
9,5\end{array}$ & $\begin{array}{l}85,2 \\
80,6 \\
80,0 \\
76,7 \\
71,0 \\
58,5 \\
43,5\end{array}$ & $\begin{array}{l}90,2 \\
87,8 \\
88,6 \\
85,8 \\
85,0 \\
78,4 \\
65,3\end{array}$ & $\begin{array}{l}54,28 \\
56,99 \\
54,13 \\
59,71 \\
55,05 \\
59,36 \\
62,69 \\
55,80\end{array}$ & $\begin{array}{r}8,89 \\
7,52 \\
8,82 \\
8,50 \\
8,51 \\
9,36 \\
10,66 \\
8,58\end{array}$ & $\begin{array}{l}3,45 \\
3,20 \\
4,22 \\
4,62 \\
4,05 \\
3,00 \\
3,52 \\
10,84\end{array}$ & $\begin{array}{l}33,38 \\
32,29 \\
32,83 \\
27,15 \\
32,39 \\
28,28 \\
23,13 \\
24,78\end{array}$ \\
\hline
\end{tabular}

* Berechnet mit der Formel $-\left[\mathrm{CO}-\mathrm{NH}-\left(\mathrm{CH}_{2}\right)_{6}-\mathrm{NH}-\mathrm{CO}-\mathrm{O}-\left(\mathrm{CH}_{2}\right)_{4}-\mathrm{O}\right]-$

Die in Tabelle 3 zutage tretenden Unterschiede in den leichteren gasförmigen Pyrolyseprodukten sind mit deren strukturellen Eigentümlichkeiten verbunden. Da die näheren Angaben über ihre Struktur fehlen, fällt es schwer, die Unterschiede in der thermischen Zersetzung mit strukturellen Unterschieden zu verbinden. Laut Literaturangaben ist über Dorlastan und Lycra bekannt, daß das erstere ein Polyesterurethan-Elastomer, 
das zweite dagegen ein Polyätherurethan-Elastomer ist $\left[{ }^{1}\right]$. Die entsprechenden Unterschiede kommen auch in ihrem thermischen Zerfall zur Geltung; so sind z. B. die CO-Gehalte entsprechend 9,1 und $21,8 \%$. Uber die übrigen Elastomere fehlen die Angaben.

In denselben Fasern wurde durch Elementaranalyse der Gehalt an Kohlenstoff, Wasserstoff und Stickstoff bestimmt. Bei der Bestimmung des Stickstoffes wurde die Methode von Dumas angewandt, der Sauerstoff dagegen wurde aus der Differenz berechnet. Der Gehalt an C und H wich im allgemeinen von der auf Grund der klassischen Polyurethanformel errechneten Elementarzusammensetzung verhältnismäßig wenig ab. Der Stickstoffgehalt aber war im Vergleich zum errechneten bedeutend kleiner. Der Stickstoffgehalt wurde auch mit Hilfe der Bestimmungsmethode von Kjeldahl geprüft. Die Resultate waren nach Kjeldahl etwas kleiner als nach Dumas. Auf Grund der Analyseangaben kann vermutet werden, daß der Stickstoffgehalt in den Molekeln der Polyurethan-Elastomere niedriger und der Sauerstoffgehalt höher ist als der errechnete (s. Tab. 3), was auch den höheren Anteil der leichteren gasförmigen Sauerstoffverbindungen (CO und $\mathrm{CO}_{2}$ ) an den Pyrolyseprodukten bedingt.

Die Unterschiede in den leichteren gasförmigen Produkten $\left(\mathrm{CO}, \mathrm{CO}_{2}\right.$, $\mathrm{CH}_{4}, \mathrm{C}_{2} \mathrm{H}_{6}$ ) der thermischen Zersetzung der Polyurethan-Elastomere können in einem gewissen Umfang zu deren gegenseitiger Identifizierung benutzt werden. Erschwert wäre bloß die Identifizierung von Spanzelle, Chemstrand und Dorlastan, da ihre CO-Gehalte innerhalb relativ enger Grenzen variieren.

Die Chromatogramme der'mit einer DMS erhaltenen Pyrolyseprodukte von Polyurethan-Elastomeren finden sich auf Abb. 2. An Hand der Chromatogramme wurden die größeren Peaks durch Vergleich der Retentionszeiten mit denen der Etalonstoffe identifiziert. Tabelle 4 enthält das Verzeichnis der identifizierten Peaks.

Gehalt an leichteren Kohlenwasserstoffen in den Pyrolyseprodukten

Tabelte 4

\begin{tabular}{|c|c|c|c|c|c|c|c|c|c|c|c|c|c|c|}
\hline & 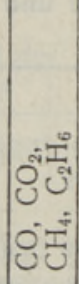 & $\frac{5}{9}$ & 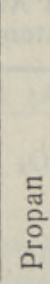 & 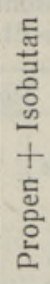 & 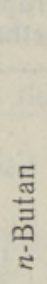 & 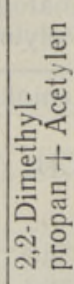 & 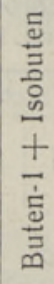 & 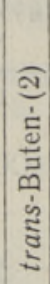 & 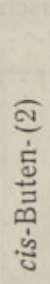 & 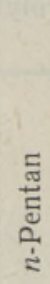 & 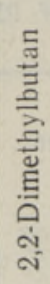 & 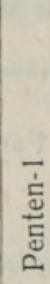 & 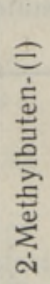 & 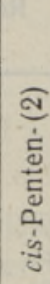 \\
\hline Nummer des Peaks & 1 & 2 & 3 & 4 & 5 & 6 & 7 & 8 & 9 & 10 & 11 & 12 & 13 & 14 \\
\hline Fr. elastomère & & + & t- & + & 0 & & + & + & 0 & + & + & 0 & & 0 \\
\hline Spanzelle & & & & & 0 & & & + & 0 & 0 & + & & & 0 \\
\hline Chemstrand & & + & + & + & 0 & $\div$ & + & 0 & 0 & 0 & + & & & 0 \\
\hline Dorlastan & & + & + & + & 0 & 0 & + & 0 & 0 & 0 & + & + & 0 & 0 \\
\hline Glanzstoffe & & + & + & + & 0 & + & + & 0 & 0 & 0 & + & & & 0 \\
\hline Fabelta & & & + & + & 0 & 0 & & 0 & 0 & 0 & + & 0 & + & 0 \\
\hline Lycra & t. & + & + & & + & 0 & & 4 & 0 & + & & 0 & & \\
\hline
\end{tabular}

$0-$ Gehalt im Vergleich zu den anderen Komponenten gering.

Der erste Peak der Chromatogramme enthält mit der Kieselgelsäule abgesonderte Komponenten (Abb. 1, Tab. 3). Von den mit der DMS-Säule abgesonderten Komponenten bilden Athen, Propan, Propen + Isobuten, $n$-Butan und in einigen Fällen Buten-1 + Isobuten die größten Peaks. 
Die genannten Stoffe sind für alle betrachteten Polyurethan-Elastomere charakteristisch. Der Anteil der anderen in Tabelle 4 aufgezählten Komponenten ist relativ geringer, falls wir von einigen Ausnahmen absehen, wie z. B. Lycra, bei deren thermischen Zersetzung im Vergleich zu den anderen Faserstoffen mehr n-Butan entsteht (Peak 5). Stickstoffhaltige Verbindungen wurden in den Chromatogrammen nicht identifiziert. Die DMS-Säule gibt im Vergleich zur SGSäule (Kieselgelsäule) charakteristischere Resultate, da die Etalonchromatogramme es

Abb. 1. Chromatogramme der Pyrolyseprodukte der Polyurethan-Elastomere, abgesondert mit der Kieselgelsäule.

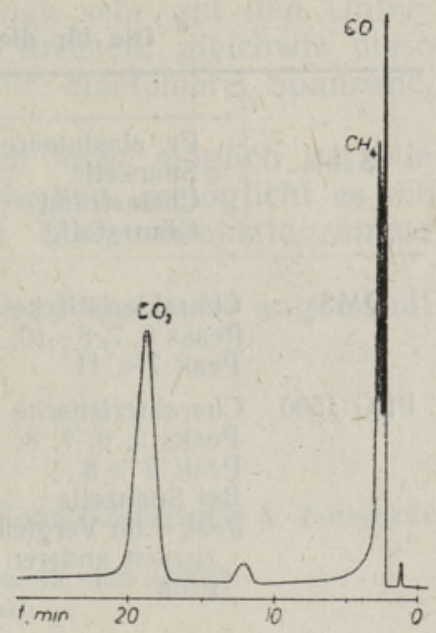

ermöglichen, unter den betrachteten Elastomeren drei Elastomere direkt zu bestimmen. Die übrigen vier haben ähnliche Chromatogramme (Abb. 2, Tab. 5) und bedürfen einer detaillierteren chromatographischen Analyse.

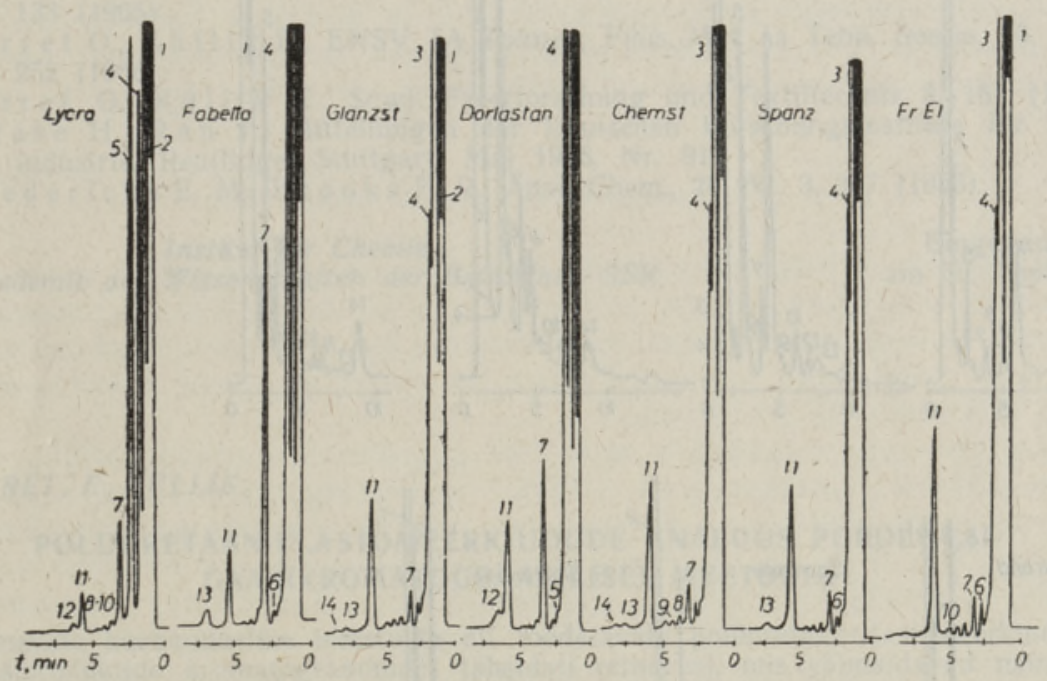

Abb. 2. Chromatogramme der Pyrolyseprodukte der Polyurethan-Elastomere, abgesondert mit der DMS-Säule.

Die Chromatogramme der mit der PEG 1500-Säule erhaltenen Pyrolyseprodukte finden sich auf Abb. 3. Die Pyrolyseprodukte wurden nicht identifiziert, da die Chromatogramme bloß zur Bestimmung der Polyurethan-Elastomere benötigt wurden. Mit der PEG 1500-Säule wurden mehr als 20 verschiedene Komponenten abgesondert, wovon die Chromatogramme den charakteristischsten Teil widerspiegeln. Bei der pyrolytischgaschromatographischen Analyse der Polyurethan-Elastomere zeitigte die PEG 1500-Säule die besten Ergebnisse (Abb. 3, Tab. 5); allerdings war es auch hier nicht möglich, die vier strukturähnlichen Elastomere von- 
Die für die Polyurethane charakteristischen Peaks

\begin{tabular}{l|l|l|l|l}
\hline \multirow{4}{*}{ Säule } & \multicolumn{3}{|c}{ Faser } \\
\cline { 2 - 5 } & $\begin{array}{l}\text { Fr. elastomère } \\
\begin{array}{l}\text { Spanzelle } \\
\text { Chemstrand } \\
\text { Glanzstoffe }\end{array}\end{array}$ & Dorlastan & Fabelta & Lycra \\
\hline
\end{tabular}

DMS Charakteristische Charakteristischer Peaks $6,7,8-10,11$ Peak 12

Peak $7<11$ Peak $7>11$

PEG 1500 Charakteristische Charakteristische Peaks 5, 6, 7, 8, 14 Peaks $7,8,10,11$ Peak $7>8$ Bei Spanzelle Peak 6 im Vergleich zu den anderen groß
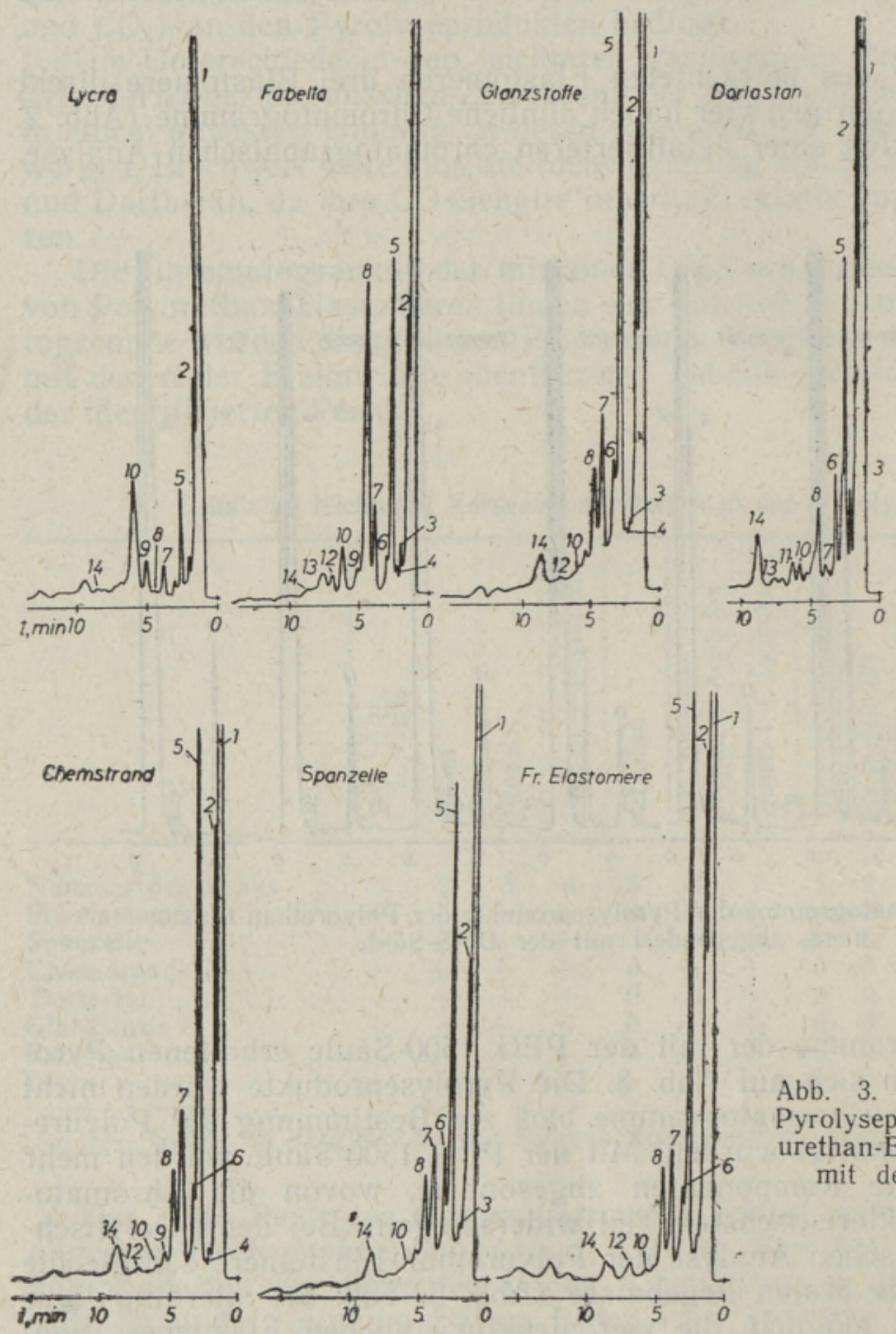
Peak $7<8$
Peak $7 \gg 11$
Peak $5 \approx 8 \quad$ Peak 8 sehr klein,

und $7<8 \quad$ Peak 10 gro $B$
Peaks 4 und 5 groß, Peak 11 klein 
einander zu unterscheiden, wohl aber konnte man sehr gut den Unterschied zwischen Dorlastan, Fabelta und Lycra machen, gleichwie diese Faserstoffe von der Gruppe zu trennen, zu der Fr. elastomère, Spanzelle, Chemstrand und Glanzstoffe gehören.

Obgleich es pyrolytisch-gaschromatographisch nicht möglich ist, alle Polyurethan-Elastomere voneinander zu unterscheiden, ermöglicht es die Methode, kleine Fasermengen mit Hilfe ihrer Etalonchromatogramme schnell miteinander zu vergleichen.

Die gleiche Methode kann bei der Analyse aller Faserstoffe angewandt werden.

\section{I T E R A T UR}

1. Heidemann G., Jellinek G., Ringens W., Kolloid-Zeitschrift \& Zeitschrift für Polymere, 221, Nr. 2, 119 (1967).

2. Mckinney R. W., J. of Gas Chromatography, 2, No. 12, 432 (1964).

3. Cox B. C., E 11 is B., Anal. Chem., 36, No. 1, 90 (1964).

4. Vo ig t J., Kunststoffe, 51, Nr. 1, 18 (1961).

5. Voigt J., Kunststoffe, 5i, Nr. 6, 314 (1961).

6. Zula ic a J., Gui och on G., Anal. Chem., 35, Nr. 11, 1724 (1963).

7. Zula ic a J., Gui o ch on G., Bull. Soc. Chim. France, No. 4, 1343 (1966).

8. Zula ic a J., Gui och on G., Bull. Soc. Chim. France, No. 4, 1351 (1966).

9. Kirret O., Küllik E., ENSV TA Toimet., Füüs.-Mat. ja Tehn. Seeria, 13, Nr. 1, 15 (1964).

10. Küllik E., Kirret O., ENSV TA Toimet., Füüs.-Mat. ja Tehn. Seeria, 14, Nr. 1, $133(1965)$

11. Kirret O., Küllik E., ENSV TA Toimet,, Füüs.-Mat. ja Tehn. Seeria, 15, Nr. 2, $252(1966)$.

12. Kirret O., Küllik E., Sowj. Faserforschung und Textiltechnik 3, 162 (1964)).

13. $\mathrm{H}$ a a se H., R a u J., Mitteilungen der Deutschen Forschungsinstitute für Textilindustrie, Reutlingen-Stuttgart, Mai 1966, Nr. 91.

14. Fredericks E. M., Brooks F. R., Anal. Chem., 28, Nr. 3, 297 (1956).

Institut für Chemie

der Akademie der Wissenschạten der Estnischen SSR

Eingegangen

am 30. Sept. 1968

O. KIRRET, E. KOLLIK

\section{POLUURETAAN-ELASTOMEERKIUDUDE ANALUUS PÜROLUUSI- GAASIKROMATOGRAAFILISEL MEETODIL}

Erinevate kaubanduslike nimetuste all toodetavate polüuretaan-elastomeerkiudainete pürolüüsiproduktide kromatogrammides täheldati erinevusi, mis vōimaldavad mōningaid vaadeldud polüuretaan-elastomeerkiudaineid üksteisest eristada.

\section{О. КИРРЕТ, Э. КЮЛЛИК}

\section{АНАЛИЗ ПОЛИУРЕТАНОВЫХ ЭЛАСТИЧЕСКИХ ВОЛОКОН МЕТОДОМ ПИРОЛИЗНОИ ГАЗОВОИ ХРОМАТОГРАФИИ}

Методом пиролизной газовой хроматографии были изучены полиуретановые эластические волокна, выпускаемые промышленностью под разными торговыми названиями. Полученные хроматограммы продуктов пиролиза имеют отличия, позволяющие различать друг от друга некоторые полиуретановые эластические волокна. 